\title{
Comparison of laser speckle contrast imaging with laser Doppler for assessing microvascular function
}

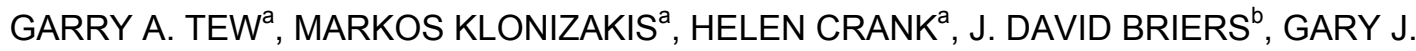 \\ HODGES $^{c}$ \\ ${ }^{a}$ Centre for Sport and Exercise Science, Sheffield Hallam University, UK \\ ${ }^{b}$ Emeritus Professor, Kingston University, UK \\ 'Department of Kinesiology, The University of Alabama, USA
}

Corresponding author:

Garry A. Tew, PhD

Centre for Sport and Exercise Science

Sheffield Hallam University

A125 Collegiate Hall

Sheffield, S10 2BP, UK

Phone: +44 (0)114225 2358

Fax: +44 (0)114225 4341

Email: g.tew@shu.ac.uk 


\section{Abstract}

Objective: To compare the inter-day reproducibility of post-occlusive reactive hyperaemia (PORH) and sympathetic vasomotor reflexes assessed by single-point laser Doppler flowmetry (SP-LDF), integrating-probe LDF (IP-LDF) and laser speckle contrast imaging (LSCI), and the spatial variability of PORH assessed by IP-LDF and LSCI. We also evaluated the relationship between IP-LDF and LSCI perfusion values across a broad range of skin blood flows.

Methods: Eighteen healthy adults (50\% male, age $27 \pm 4$ years) participated in this study. Using SPLDF, IP-LDF and LSCI, an index of skin blood flow was measured on the forearm during PORH (1-, 5and 10-min occlusions) and on the finger pad during inspiratory gasp and cold pressor tests. These tests were repeated 3-7 days later. Data were converted to cutaneous vascular conductance (CVC; laser Doppler flow/mean arterial pressure) and expressed as absolute and relative changes from prestimulus $C V C\left(\triangle C V C_{A B S}\right.$ and $\triangle C V C_{R E L}$, respectively), as well as normalised to peak CVC for the PORH tests. Reproducibility was expressed as within-subjects coefficients of variation (CV, in \%) and intraclass correlation coefficients.

Results: The reproducibility of PORH on the forearm was poorer when assessed with SP-LDF and IPLDF compared to LSCI (e.g., CV for 5-min PORH $\triangle C V_{A B S}=35,27$ and 19\%, respectively), with no superior method of data expression. In contrast, the reproducibility of the inspiratory gasp and cold pressor test responses on the finger pad were better with SP-LDF and IP-LDF compared to LSCI (e.g., $C V$ for inspiratory gasp $\Delta C V C_{R E L}=13,7$ and $19 \%$, respectively). The spatial variability of $P O R H$ responses was poorer with IP-LDF compared to LSCI (e.g., CV ranging $11-35 \%$ versus $3-16 \%$, respectively). The association between simultaneous $\mathrm{LSCl}$ and IP-LDF perfusion values was nonlinear.

Conclusion: The reproducibility of cutaneous PORH was better when assessed with LSCI compared to SP-LDF and IP-LDF; probably due to measuring larger skin areas (lower inter-site variability). However, when measuring sympathetic vasomotor reflexes on the finger pad, reproducibility was better with SP-LDF and IP-LDF, perhaps due to the high sensitivity of LSCI to changes in skin blood flow at low levels.

Keywords: LASCA, laser Doppler flowmetry, reliability, blood flow, reactivity, skin 


\section{Introduction}

Laser Doppler flowmetry (LDF) and laser Doppler imaging (LDI) are techniques that use the optical Doppler effect to estimate changes in skin blood flow (SkBF) non-invasively. When combined with specific reactivity tests, these techniques can be used to assess skin microvascular reactivity. For example, the increase in SkBF above baseline following the release of a brief arterial occlusion, commonly known as post-occlusive reactive hyperaemia $(\mathrm{PORH})$, is often used as a test of skin microvascular vasodilator function (Cracowski et al., 2006), and the reflex skin vasoconstrictor response to an inspiratory gasp or cold pressor test is often used as a measure of autonomic sympathetic function (Aso et al., 1997, Young et al., 2006, Bornmyr et al., 1999, Asahina et al., 2008). These tests have been used as clinical surrogate markers in many diseases, including diabetes (Yamamoto-Suganuma and Aso, 2009, Bornmyr et al., 1997), chronic kidney disease (Rossi et al., 2008), and peripheral arterial disease (Nukada et al., 1998). However, lack of standardisation in data expression and issues surrounding poor reproducibility have limited the use of these tests in routine clinical practice.

Single-point LDF probes, which are commonly used in the assessment of skin microvascular reactivity, appear prone to large variability in recordings. For example, Roustit et al. (Roustit et al., 2010a, Roustit et al., 2010b) demonstrated that the inter-day reproducibility of PORH assessed at the forearm with single-point LDF (SP-LDF) is poor, with within-subjects coefficients of variation $\geq 30 \%$. This finding was thought to be related to the small measurement volume of single-point probes $(<1$ $\mathrm{mm}^{3}$ (O'Doherty et al., 2009)) and the assumed large spatial heterogeneity in capillary density in forearm skin (Johnson et al., 1984, Wardell et al., 1994). Therefore, inter-site and inter-day reproducibility might be improved by evaluating and averaging SkBF responses over larger areas. Integrating LDF probes integrate the SkBF signal from several optical fibres, which theoretically should reduce the variability by a factor equal to the square root of the number of fibres (Wahlberg and Fagrell, 1994). Indeed, we recently presented integrating-probe LDF (IP-LDF) data that, when compared with the SP-LDF data of Roustit et al. (Roustit et al., 2010b), had superior reproducibility for assessing cutaneous thermal hyperaemia (Tew et al., 2011). Along this line, LDI could also be used to evaluate SkBF over larger areas; however, the image update rates of LDI systems are currently too slow to accurately assess the kinetics of PORH and sympathetic vasomotor reflexes, which limits the interest, i.e., full-field real-time evaluation of SkBF.

Another non-invasive laser-based method for estimating blood flow, laser speckle contrast imaging (LSCI), is gaining increased interest as an alternative to LDF and LDI for assessing skin microvascular function (Roustit et al., 2010b, Mahe et al., 2011, Rousseau et al., 2011). This technique is based on the same fundamental principle as LDF/LDI, but with a different and faster way of signal processing that allows full-field imaging of skin perfusion in near real time (Briers, 2007, Draijer et al., 2009, Boas and Dunn, 2010). Roustit et al. (Roustit et al., 2010b) recently tested one of the few commercially-available speckle imagers, the PeriCam PSI System from Perimed, and reported that the reproducibility of PORH was better with LSCI compared to SP-LDF, probably due to lower inter-site variability when measuring larger skin areas. However, it is unknown if LSCI has superior reproducibility to IP-LDF. Further research is also needed to describe and compare the 
reproducibility of other microvascular reactivity tests (e.g., sympathetic vasomotor reflexes) assessed with SP-LDF, IP-LDF and LSCI.

For the purposes of physiological research and clinical diagnostics it is preferred that the perfusion signal, be it from LDF, LDI or LSCI is linearly related with SkBF. For low blood concentrations, the concentration of red blood cells and their average velocity are both linearly related by the perfusion estimate given by laser Doppler methods (Bonner and Nossal, 1981). However, speckle contrast flowmetry modelling has, to date, mostly focussed on retrieving velocity information of scattering particles rather than their flux which includes concentration (Briers and Webster, 1995, Ramirez-San-Juan et al., 2008, Parthasarathy et al., 2008), and the relationship between speckle contrast and velocity is non-linear (Draijer et al., 2009). A rigorous theory linking the statistics to perfusion does not yet exist. Therefore, for new $\mathrm{LSCl}$ instruments that purport to measure skin perfusion, it appears important to evaluate: (i) if the linear output range operates within the physiological parameters of the tissue(s) of interest, and; (ii) if the LSCl-derived perfusion signal is sensitive to changes in both the concentration of red blood cells and their average velocity.

The primary aim of the present study was to compare the inter-day reproducibility of PORH and sympathetic vasomotor reflexes (inspiratory gasp and cold pressor tests) assessed by SP-LDF, IP-LDF and LSCI. We hypothesised that the inter-day reproducibility of these skin microvascular reactivity tests would be best when assessed using $\mathrm{LSCl}$ and worst with SP-LDF. Secondary objectives were to compare the spatial variability of PORH assessed using IP-LDF and LSCI, to identify the most reproducible form of data expression, to evaluate the relationship between IP-LDF and LSCI perfusion values across a broad range of SkBFs, and to investigate if this relationship was dependent on whether perfusion changes were concentration- or velocity-dependent.

\section{Methods}

Participants

With local Research Ethics Committee approval, 18 healthy adults were recruited (50\% male, age $27 \pm 4$ years). Individuals with any significant medical history were excluded, along with smokers and people taking any form of medication except oral contraceptives. All volunteers provided written, informed consent prior to participation. This study was carried out in accordance with the Declaration of Helsinki.

\section{Protocol}

Participants were instructed to abstain from vigorous exercise within 24 hours and food and caffeine within 2 hours of testing. All microvascular tests were performed in a temperature-controlled room $\left(23 \pm 1{ }^{\circ} \mathrm{C}\right)$ with participants resting in a comfortable semisupine position. After an acclimatisation period of $>20 \mathrm{~min}$, SkBF responses at rest and during inspiratory gasp, cold pressor, and PORH tests were assessed simultaneously by SP-LDF, IP-LDF and LSCI (Test 1). This sequence was repeated 3-7 days later (Test 2). Heart rate and systolic, diastolic, and mean arterial pressures were recorded intermittently from the right arm (Dinamap Dash 2500, GE Healthcare, USA) at baseline and during the 
reactivity tests. To obtain reproducibility data in the conditions of routine use, the locations of SkBF sites were not marked and the menstrual phase of the female participants was not standardised.

Two finger pads were randomly selected from the index, middle, and ring fingers of the left hand. SkBF was measured as cutaneous red blood cell flux at both of these sites by laser Doppler flowmetry (Periflux System 5000, Perimed, Järfälla, Sweden; $5 \mathrm{~Hz}$ sampling rate); one finger pad was instrumented with a single-point probe that had an integrated heater (Probe 457, Perimed) and the other a 7-point integrating probe (Probe 413, Perimed) that was housed in a heating disc (Model 455, Perimed). The heaters of both LDF probes were set at $33{ }^{\circ} \mathrm{C}$ (thermoneutral). The remaining finger was used for $\mathrm{LSCl}$ measures of finger pad SkBF (PeriCam PSI System, Perimed), with the laser head placed $14 \mathrm{~cm}$ above the skin and an image acquisition rate of $3 \mathrm{~Hz}$. After a 5-min baseline recording, participants were instructed to take a rapid, deep breath inwards and hold for 10 $s$ before breathing normally. This procedure was completed twice more at 2-min intervals. After return of SkBF to baseline ( $\sim 5 \mathrm{~min})$, the cold pressor test was performed by placing the right hand in cold water $\left(\sim 4{ }^{\circ} \mathrm{C}\right)$ for $60 \mathrm{~s}$.

After the sympathetic vasomotor reflex tests the LDF probes and LSCl field of interest were moved to the left forearm. An additional integrating LDF probe was placed $\sim 5$ $\mathrm{cm}$ from the other to allow for the assessment of spatial variability. After a 5 -min baseline recording, blood flow was occluded for 1 min by inflating a cuff placed on the upper left arm to $200 \mathrm{mmHg}$. This was followed by a 5 -min post-occlusion period during which PORH was recorded (Mahe et al., 2011). The PORH test was completed two more times, first with a 5min occlusion, and then a 10-min occlusion. This allowed assessment of the linearity of the LSCI-LDF perfusion relationship over a broad range of SkBFs.

Data analysis

Data were stored on a computer and analysed off-line with signal processing software (PeriSoft 2.5.5 for LDF measurements and PimSoft 1.3 for LSCI measurements; Perimed). Measurements of SkBF (recorded in arbitrary perfusion units, APU) were divided by mean arterial pressure (in $\mathrm{mmHg}$ ) to give cutaneous vascular conductance (CVC) in APU/mmHg. The vasoconstrictor responses to inspiratory gasp and contralateral hand cooling were expressed as absolute and relative (\%) changes from pre-stimulus $C V C$ ( $\triangle C V C_{A B S}$ and $\triangle C V C_{R E L}$, respectively). The amplitudes of the PORH responses were expressed as absolute and relative (\%) changes from prestimulus $C V C\left(\triangle C V C_{A B S}\right.$ and $\triangle C V C_{R E L}$, respectively), and peak as a percentage of $C V C$ after the 10min occlusion (\%CVC $\mathrm{CEAK}_{\text {). }}$

For the LSCl analyses, circular regions of interest of $\sim 80 \mathrm{~mm}^{2}$ were used (Roustit et al., 2010b): one for the finger pad measures, and two adjacent to the integrating LDF probes for the forearm measures. SkBF data were averaged over $3 \mathrm{~min}$ for the baseline periods, $30 \mathrm{~s}$ for the occlusion periods, and $3 \mathrm{~s}$ for the PORH peak and the nadirs of the sympathetic vasomotor reflex responses. 
Statistical analysis

Data are expressed as the means $\pm S D$, unless otherwise stated. Inter-day reproducibility was expressed as within-subject coefficients of variation (CV) and intra-class correlation coefficients (ICC). The CV values were calculated using the root mean square method (Gluer et al., 1995), with $\leq 10 \%$, $10-25 \%$ and $\geq 25 \%$ considered good, moderate and poor reproducibility, respectively (Tew et al., 2011). The ICC values and accompanying confidence intervals were assessed using the two-way mixed model approach, with $<0.40,0.40-0.75$ and $>0.75$ considered as poor, fair-to-good and excellent reproducibility, respectively (Landis and Koch, 1977). The spatial (inter-site) variability of PORH responses assessed with IP-LDF and LSCI was also calculated using within-subject CVs (i.e., we evaluated the variability of measures between forearm sites 1 and 2 for both techniques).

The relationship between LSCI and IP-LDF measures of forearm cutaneous perfusion was evaluated by fitting linear and power models to the data (baseline, occlusion, and 1-, 5- and 10-min $\mathrm{PORH}$ responses) and comparing the sum of the squared residuals for each model. We further evaluated the linearity of the LSCI perfusion signal by comparing the strength of the LSCI-LDF relationship between raw and log-transformed data using Pearson's correlation tests. Finally, we investigated if the relationship between LSCl- and LDF-derived measures of perfusion was dependent on whether perfusion changes were concentration- or velocity-dependent by determining the relationship (Pearson's test) between the LDF-based concentration-velocity slopes and the LSCI-LDF perfusion slopes. Statistical analyses were performed using Predictive Analytics SoftWare Statistics v18.0 (SPSS: An IBM Company, New York, USA) and significance was set at $P<0.05$.

\section{Results}

Test-retest CVC data obtained by SP-LDF, IP-LDF and LSCI are presented in Tables 1, 2 and 3 , respectively. The vasoconstrictor response to inspiratory gasp and contralateral hand cooling was absent in two of the female participants; therefore, the analyses for these reactivity tests are based on $n=16$. The CVC responses for each test did not differ significantly between males and females (data not presented); therefore, male and female data were pooled. The peak and duration of the PORH response was proportional to the duration of the occlusion in all participants. For example, the $\triangle \mathrm{CVC}_{\mathrm{REL}}$ responses determined by LSCI on Test 1 were $166 \pm 65 \%, 243 \pm 74 \%$ and $316 \pm 87 \%$ for 1 -, 5- and 10-min occlusions, respectively.

Inter-day reproducibility of cutaneous microvascular perfusion responses assessed by SP-LDF Inter-day reproducibility data for SP-LDF are presented in Table 1 . The reproducibility of forearm baseline responses was poor when expressed as CV (25-32\%) and fair-to-good when expressed as ICC (0.58-0.59). This was also the case for finger pad baseline responses (CV $=52 \%$, ICC $=0.44)$. The PORH responses generally had poor reproducibility for all methods of data expression (Table 1). The inspiratory gasp and cold pressor responses had moderate-to-good reproducibility when expressed as $\triangle C V C_{R E L}$ and poor-to-moderate reproducibility when expressed as $\triangle \mathrm{CVC}_{\mathrm{ABS}}$ (Table 1). 
Inter-day reproducibility of cutaneous microvascular perfusion responses assessed by IP-LDF

As for SP-LDF, the reproducibility of forearm and finger pad baseline responses was poor when expressed as CV (33-44\%) and fair-to-good when expressed as ICC (0.59-0.65) (Table 2). The PORH responses had poor reproducibility when expressed as $\mathrm{CV}$ and fair-to-good reproducibility when expressed as ICC, with no clearly superior method of data expression (Table 2). The inter-day reproducibility of the inspiratory gasp and cold pressor responses was good when expressed as $\triangle C V C_{R E L}$ and poor-to-moderate reproducibility when expressed as $\Delta C C_{A B S}$ (Table 2).

Inter-day reproducibility of cutaneous microvascular perfusion responses assessed by LSCI

Baseline responses had moderate-to-good reproducibility on the forearm (CV $=16 \%$, ICC $=$ 0.68-0.76) and poor-to-fair reproducibility on the finger pad (CV $=46 \%, I C C=0.51)$ (Table 3). The PORH responses generally had moderate-to-good reproducibility, with $\triangle C V C_{A B S}$ and $\% C V C_{P E A K}$ being better than $\triangle C V C_{\text {REL }}$ (Table 3). The reproducibility of the inspiratory gasp and cold pressor responses was moderate when expressed as $\triangle C V C_{R E L}$ and poor-to-moderate reproducibility when expressed as $\triangle \mathrm{CVC}_{\mathrm{ABS}}$ (Table 3).

Spatial variability of PORH responses simultaneously assessed with IP-LDF and LSCI

Spatial variability data for IP-LDF and LSCI are presented in Table 4. The inter-site reproducibility of baseline responses was poor when assessed by IP-LDF (CV $=29-35 \%$ ), and moderate-to-good when assessed by LSCI (CV = 13-15\%). The inter-site reproducibility of PORH responses was also better when assessed by LSCI compared to IP-LDF, with CVs ranging 3-16\% and $11-37 \%$, respectively. The 5 -min PORH response expressed as $\% \mathrm{CVC}_{\text {PEAK }}$ had the best inter-site reproducibility (CV $=11 \%$ and $3 \%$ for IP-LDF and LSCI, respectively).

\section{Relationship between LSCI and IP-LDF measures of forearm cutaneous perfusion}

The sum of the squared residuals for the linear and power functions was 4.6 and 2.9, respectively, indicating that the power function fitted the data better than the linear function. The power function was described by the following equation: $y=0.7177 x^{1.6027}$. Other evidence supporting a non-linear relationship between the LSCI signal and LDF-derived measures of SkBF include: (i) the line of best-fit for the linear model in Fig. 1 crossed the ordinate at -0.2513 , and; (ii) the LSCI-LDF perfusion relationship was stronger for log-transformed data $(r=0.966)$ than raw data $(r=0.925)$, although both were highly significant $(P<0.001)$. Finally, there was a trend towards a moderate positive relationship between the LDF-based concentration-velocity slopes and the LSCI-LDF perfusion slopes ( $r=0.412, P=0.089$; Fig. 2 ), suggesting that $\mathrm{LSCl}$ becomes less sensitive to changes in SkBF when these changes are more dependent on red blood cell concentration than red blood cell velocity.

\section{Discussion}


The main findings from this study are as follows: (i) the inter-day reproducibility of forearm PORH was better when assessed with LSCI compared to SP-LDF and IP-LDF; (ii) the inter-day reproducibility of the inspiratory gasp and cold pressor test responses on the finger pad were better with SP-LDF and IP-LDF compared to LSCI; (iii) the relationship between LSCI and IP-LDF measures of forearm cutaneous perfusion was non-linear, and; (iv) the LSCI perfusion signal was more sensitive to changes in red blood cell velocity than red blood cell concentration.

Reproducibility of skin microvascular reactivity assessed using SP-LDF, IP-LDF and LSCI

Skin microvascular reactivity is commonly assessed on the forearm using SP-LDF coupled with PORH. However, our findings (Table 1) and those of others (Roustit et al., 2010a, Roustit et al., $2010 b$ ) indicate poor inter-day reproducibility of this test, which is probably related to the assumed large spatial heterogeneity in forearm capillary density (Johnson et al., 1984, Wardell et al., 1994). The issue of spatial variability might be minimised by measuring SkBF over a larger area with IP-LDF or LSCI. Indeed, our results indicate that reproducibility is better for both these techniques, but is only acceptable with LSCI. The superior reproducibility of LSCI compared to IP-LDF is probably explained by its larger measurement area and better inter-site reproducibility (Table 4). We are only aware of one other study that has assessed the reproducibility of forearm PORH using LSCl. Roustit et al. (Roustit et al., 2010b) reported lower CVs for data expressed as $\Delta C V C_{A B S}$ and $\Delta C V C_{R E L}$ (11 versus $19 \%$ and 14 versus $22 \%$, respectively). These differences might be explained by the fact that we did not standardise baseline skin temperature, an approach which has been demonstrated to enhance the reproducibility of forearm PORH assessed by SP-LDF (Roustit et al., 2010a, Roustit et al., 2010b). Roustit et al. (Roustit et al., 2010b) used a water-filled heating probe for this purpose; this was not available for the present study.

In contrast to our hypothesis and the findings for forearm $\mathrm{PORH}$, the inter-day reproducibility of the inspiratory gasp and cold pressor test responses on the finger pad was better with SP-LDF and IP-LDF compared to LSCI. This is probably related to the observed relationship between the LSCI signal and SkBF (assessed by IP-LDF), which over a broad range of flows appeared curvi-linear (Fig. 1). Data for the sympathetic vasomotor reflex tests are not presented in Fig. 1, but they would be clustered between the occlusion and baseline data on the bottom-left part of the chart. Here, a small change in SkBF, as may be expected between day-to-day measurements, would result in a relatively large change in the LSCI signal. This might explain the relatively large variability in LSCI perfusion values for these provocations between Tests 1 and 2 . This finding might also be partly explained by the fact that we did not standardise skin temperature for the LSCI-based measurements. Again, we did not have a heating probe to use for this purpose; however, it is important to note that the heating probe used by Roustit et al. (Roustit et al., 2010b) is too large for use on the finger pad.

Our findings allow the estimation of the number of participants that would be required to detect a biologically meaningful difference in forearm PORH using SP-LDF, IP-LDF and LSCI. For example, when data are expressed as $\triangle \mathrm{CVC}_{\mathrm{ABS}}$, and using the 5-min $\mathrm{PORH}$ data from Test 1 with $\alpha=0.05$ and $80 \%$ power, 31,34 and 16 participants would be needed to detect a $20 \%$ change using each technique, respectively. Similarly, for the inspiratory gasp test data expressed as $\triangle C V C_{R E L}, 10,8$ 
and 9 participants would be needed with each technique, respectively. Finally, for the cold pressor test data expressed as $\Delta \mathrm{CVC}_{\mathrm{REL}}, 8,8$ and 12 participants would be needed with each technique, respectively.

Technical issues surrounding the use of LDF and LSCI to measure SkBF

One problem of LSCl compared with LDF is its apparent lack of linearity with perfusion. To explore this further, we need to look at the fundamental theory of the Doppler and speckle contrast techniques. Both analyse the intensity fluctuations observed when laser light is scattered from a medium containing moving scatterers, but the theoretical approach to the two techniques is different.

The Doppler explanation of the phenomenon is that light scattered from moving objects has its frequency shifted by the Doppler effect. Because the frequencies of light waves are so high, the only way to observe the frequency shift is to combine it with an unshifted frequency. (This can be a local reference beam, or light scattered from stationary scatterers in the field.) The two frequencies produce a beat frequency equal to the difference between the two frequencies.

The explanation of laser speckle is that it is a random interference effect between waves of the same frequency. With all the scatterers stationary, at any point in the speckle pattern the intensity will be constant. If some of the particles move, the intensity at any point in the speckle pattern will fluctuate as the relative path lengths from the different scatterers vary. The two explanations appear to be fundamentally different, but it can easily be shown that the mathematical formulae connecting the frequency of the fluctuations and the velocity of the moving scatterers are identical. The two approaches are just two ways of looking at the same phenomenon (Briers, 1996).

In both theories, the line-of-sight velocity of the moving scatterers is directly proportional to the frequency of the fluctuations. This would suggest that both techniques are linear with respect to velocity. In the case of Doppler, however, it has been accepted for some thirty years that if you take the first moment of the power spectrum of the fluctuations, then this scales linearly with both velocity and with concentration (number of moving scatterers) (Bonner and Nossal, 1981). In the case of blood flow, this is a measure of perfusion. If a Doppler system uses this algorithm (first moment of the power spectrum), then it should be linear with respect to perfusion.

The question now arises, is the speckle contrast technique linear with respect to either velocity or perfusion? The short answer is no. The first paper published on the possible use of speckle contrast showed that a plot of speckle contrast against the logarithm of the ratio of the "correlation time" $T_{c}$ to the integration time T gives an S-shaped curve (Fercher and Briers, 1981). ( $T_{c}$ is inversely proportional to some characteristic velocity $v_{c}$ and $T$ is, of course, a constant for a single exposure.) $A$ typical curve is presented in Fig. 3. This shape of curve has been confirmed experimentally by several workers (Thompson and Andrews, 2010) and means that the relationship between speckle contrast and velocity is highly non-linear. This property of non-linearity is also supported by the data presented in this paper - Fig. 1 indicates non-linearity between the LSCI and LDF signals. This does not necessarily mean that $\mathrm{LSCl}$ is less quantitative than LDF as a means of measuring perfusion: it simply means that careful calibration and/or interpretation are necessary. 
However, although the speckle contrast is not linear with respect to velocity, reference to Fig. 3 shows that the central portion of the S-shaped curve is approximately linear over quite a large range, say a contrast range of 0.2 to 0.8 , when plotted against the logarithm of $\mathrm{T}_{\mathrm{C}} / \mathrm{T}$. This means that the speckle contrast is approximately linear with respect to relative changes in velocity. In other words, whatever the starting value of velocity, the same percentage change in velocity will produce approximately the same change in speckle contrast. $\mathrm{LSCl}$ is therefore likely to be more useful for detecting changes in perfusion rather than absolute measurements. (This argument, like Fig. 3 itself, is based on the fundamental physics that speckle contrast is a function of velocity alone: the effect of concentration, or the number of moving scatterers, is ignored. We shall return to this point later, when we discuss whether the technique measures velocity or perfusion.)

The exact form of the S-shaped curve depends on the algorithm used in the analysis. The algorithm used in the original paper (Fercher and Briers, 1981) made a lot of approximations, including an assumption that the velocity distribution was Lorentzian. (Fig. 3 assumes such a Lorentzian distribution.) There was also an error in the analysis, caused by starting from a relationship connecting the spatial variance in a time-averaged speckle pattern with the autocovariance of the temporal variations that was in common use at the time, but which was later shown to be incomplete: this error has recently been corrected (Bandyopadhyay et al., 2005). The main point, though, is the importance of choosing the correct algorithm.

A way round the problem of having to choose the correct algorithm (i.e., the correct velocity distribution) has been presented recently by Thompson and Andrews (Thompson and Andrews, 2010). They used a multiple-exposure technique, in which the exposure time is doubled between exposures. This method allowed the calculation of the first moment of the power spectrum of the fluctuations exactly the parameter that is measured in the Doppler technique. Thus there was no need to assume a velocity distribution - they were effectively measuring this. The linearity of the method would be exactly the same as the linearity of the Doppler method.

The question still remains, though, whether the speckle contrast technique is measuring velocity or perfusion. In other words, is the measurement affected by the concentration of the scatterers (red blood cells in this case)? A moment's thought shows that the speckle contrast must be affected by the number of moving scatterers involved, and hence by the concentration. Consider a speckle pattern produced only by stationary scatterers: assuming conditions are ideal, the speckle contrast will be 1 (the maximum). Now add just a few moving particles: it is clear that some intensity fluctuations will be introduced, so that a time-integrated image of the speckle pattern will show some loss of contrast. However, the intensity of each individual speckle will show only small fluctuations about its original (stationary) value. This means that the speckle pattern will be dominated by the pattern from the stationary scatterers and the loss of contrast will be small, even for long integration times. As the number of moving scatterers is increased, the effect of the stationary scatterers will diminish and, for a given integration time, the speckle contrast will decrease. The speckle contrast produced by a mixture of moving and stationary scatterers over a long integration time has been studied in earlier work (Briers, 1978), and the analysis presented there has been confirmed experimentally (Rabal et al., 2003). However, a more in-depth study of this effect is necessary. In the 
current work, we have found that changes in the LSCI signal appear to be more sensitive to changes in the velocity of red blood cells rather than to changes in their concentration. Others, however, are convinced that concentration plays a role and that LSCI measures perfusion.

This question of whether LSCl measures velocity or perfusion is still not answered. Recent papers are almost unanimous in calling for more work to be done on this (Draijer et al., 2009, Boas and Dunn, 2010, Roustit et al., 2010b). Some even cast doubt on the assumption that Doppler can produce definitive perfusion measurements (Thompson and Andrews, 2010). We join this call for more work to be done. Until we have a definitive relationship between the numbers produced by LSCI and perfusion (or velocity), we suggest that the technique is best fitted to relative measurements, especially the detection of changes, where it is a very powerful technique. As intimated by Thompson and Andrews (2010), there may also be a case for having another look at the assumptions made in the theory that links LDF and perfusion.

There is also a need to explore the relationship between LDF and LSCI in more depth - the scatterplot of Fig. 2 suggests that there is a lot of uncertainty about this relationship and Fig. 1 suggests that this is especially the case at low flow rates. Finally, the importance of using the correct algorithm when interpreting speckle contrast cannot be over-emphasized. We understand that some workers are using a perfusion index equal to the reciprocal of the contrast, or some simple function of this. From Fig. 3, this is clearly a very crude approximation. Provided the instrument is calibrated, of course, or if one merely wishes to detect changes, it does not really matter. But it is dangerous to ascribe absolute quantitative perfusion to contrast measurements, at least until a lot more theoretical and experimental work has been carried out.

\section{Summary}

We have presented data on the inter-day reproducibility of PORH and sympathetic vasomotor reflexes (inspiratory gasp and cold pressor tests) assessed by SP-LDF, IP-LDF and LSCI in healthy young adults. The reproducibility of forearm PORH was better when assessed with $\mathrm{LSCl}$ compared to SP-LDF and IP-LDF, probably due to a lower inter-site variability when measuring larger skin areas. However, this appeared not to be the case when measuring sympathetic vasomotor reflexes on the finger pad; here, reproducibility was better with SP-LDF and IP-LDF, perhaps due to a relatively high sensitivity of LSCI to changes in SkBF at low levels. There was no clearly superior method of data presentation for the PORH tests, whereas data for the inspiratory gasp and cold pressor tests should preferentially be expressed as $\triangle C V C_{R E L}$. Finally, we demonstrated that, over a large range of SkBFs, the relationship between LSCI and IP-LDF measures of forearm cutaneous perfusion was non-linear, and that the LSCI perfusion signal was more sensitive to changes in red blood cell velocity than red blood cell concentration. However, further research is needed to assess the relationship between the LSCl signal and SkBF.

\section{Disclosures}

The LSCI device was loaned by Perimed®, but the company had no involvement in any aspect of this study. 


\section{Author contributions}

GAT and GJH conceived and developed this project. GAT, MK and HC undertook the assessment sessions. GAT, MK, JDB and GJH analysed and interpreted the data. All authors contributed to and approved the final version of the manuscript.

\section{Acknowledgements}

We would like to thank the participants for their time and commitment to this study and Perimed $₫$ for loaning us the LSCI device.

\section{References}

Asahina, M., et al., 2008. Measurements of sweat response and skin vasomotor reflex for assessment of autonomic dysfunction in patients with diabetes. J Diabetes Complications, 22, 278-283.

Aso, Y., et al., 1997. Evaluation of skin vasomotor reflexes in response to deep inspiration in diabetic patients by laser Doppler flowmetry. A new approach to the diagnosis of diabetic peripheral autonomic neuropathy. Diabetes Care, 20, 1324-1328.

Bandyopadhyay, R., et al., 2005. Speckle-visibility spectroscopy: A tool to study time-varying dynamics. Rev. Sci. Instrum. 76, 093110.

Boas, D.A., Dunn, A.K., 2010. Laser speckle contrast imaging in biomedical optics. J. Biomed. Opt. 15, 011109.

Bonner, R., Nossal, R., 1981. Model for laser Doppler measurements of blood-flow in tissue. Appl. Opt. 20, 2097-2107.

Bornmyr, S., et al., 1999. Detection of autonomic sympathetic dysfunction in diabetic patients. A study using laser Doppler imaging. Diabetes Care, 22, 593-597.

Bornmyr, S., et al., 1997. Cutaneous vasomotor responses in young type I diabetic patients. J Diabetes Complications, 11, 21-26.

Briers, J.D., 1978. Statistics of Fluctuating Speckle Patterns Produced by a Mixture of Moving and Stationary Scatterers. Opt. Quantum Electron. 10, 364-366.

Briers, J.D., 1996. Laser Doppler and time-varying speckle: A reconciliation. J. Opt. Soc. Am. 13, 345350.

Briers, J.D., 2007. Laser speckle contrast imaging for measuring blood flow. Optica Applicata, 37, 139-152.

Briers, J.D., Webster, S., 1995. Quasi Real-Time Digital Version of Single-Exposure Speckle Photography for Full-Field Monitoring of Velocity or Flow-Fields. Opt. Commun. 116, 36-42.

Cracowski, J.L., et al., 2006. Methodological issues in the assessment of skin microvascular endothelial function in humans. Trends Pharmacol. Sci. 27, 503-508.

Draijer, M., et al., 2009. Review of laser speckle contrast techniques for visualizing tissue perfusion. Las. Med. Sci. 24, 639-651. 
Fercher, A.F., Briers, J.D., 1981. Flow visualization by means of single-exposure speckle photography. Opt. Commun. 37, 326-330.

Glüer, C.C., et al., 1995. Accurate assessment of precision errors: how to measure the reproducibility of bone densitometry techniques. Osteoporos. Int. 5, 262-70.

Johnson, J.M., et al., 1984. Laser-Doppler measurement of skin blood flow: comparison with plethysmography. J. Appl. Physiol. 56, 798-803.

Landis, J.R., Koch, G.G., 1977. The measurement of observer agreement for categorical data. Biometrics, 33, 159-74.

Mahe, G., et al., 2011. Laser speckle contrast imaging accurately measures blood flow over moving skin surfaces. Microvasc. Res. 81, 183-188.

Nukada, H., et al., 1998. Preservation of skin vasoconstrictor responses in chronic atherosclerotic peripheral vascular disease. Angiology, 49, 181-8.

O'Doherty, J., et al., 2009. Comparison of instruments for investigation of microcirculatory blood flow and red blood cell concentration. J. Biomed. Opt. 14, 034025.

Parthasarathy, A.B., et al., 2008. Robust flow measurement with multi-exposure speckle imaging. Opt. Express, 16, 1975-1989.

Rabal, H.J., et al., 2003. Numerical model for dynamic speckle: an approach using the movement of the scatterers. J. Opt. A-Pure Appl. Opt. 5, S381-S385.

Ramirez-San-Juan, J.C., et al., 2008. Impact of velocity distribution assumption on simplified laser speckle imaging equation. Opt. Express, 16, 3197-3203.

Rossi, M., et al., 2008. Blunted post-ischemic increase of the endothelial skin blood flowmotion component as early sign of endothelial dysfunction in chronic kidney disease patients. Microvasc. Res. 75, 315-322.

Rousseau, P., et al., 2011. Increasing the "region of interest" and "time of interest", both reduce the variability of blood flow measurements using laser speckle contrast imaging. Microvasc. Res.

Roustit, M., et al., 2010a. Reproducibility and methodological issues of skin post-occlusive and thermal hyperemia assessed by single-point laser Doppler flowmetry. Microvasc. Res. 79, 102-108.

Roustit, M., et al., 2010b. Excellent reproducibility of laser speckle contrast imaging to assess skin microvascular reactivity. Microvasc. Res. 80, 505-511.

Tew, G.A., et al., 2011. Reproducibility of cutaneous thermal hyperaemia assessed by laser Doppler flowmetry in young and older adults. Microvasc. Res. 81, 177-182.

Thompson, O.B., Andrews, M.K., 2010. Tissue perfusion measurements: multiple-exposure laser speckle analysis generates laser Doppler-like spectra. J. Biomed. Opt. 15, 027015.

Wahlberg, E., Fagrell, B., 1994. Spatial and temporal variation in laser Doppler flux values in healthy lower limbs: comparison between the standard and the multiprobe. Int. J. Microcirc. Clin. Exp. 14, 343-346.

Wardell, K., et al., 1994. Spatial heterogeneity in normal skin perfusion recorded with laser Doppler imaging and flowmetry. Microvasc. Res. 48, 26-38. 
Yamamoto-Suganuma, R., Aso, Y., 2009. Relationship between post-occlusive forearm skin reactive hyperaemia and vascular disease in patients with Type 2 diabetes--a novel index for detecting micro- and macrovascular dysfunction using laser Doppler flowmetry. Diabetic Med. 26, 83-8.

Young, T.M., et al., 2006. Skin vasomotor reflex responses in two contrasting groups of autonomic failure: multiple system atrophy and pure autonomic failure. J. Neurol. 253, 846-50.

\section{Figure legends}

Fig. 1.

Laser speckle contrast imaging (LSCl) perfusion data plotted against integrating-probe laser Doppler flowmetry (IP-LDF) perfusion data. CVC indicates cutaneous vascular conductance in APU/mmHg. Note the non-linear relationship between the different measures of cutaneous perfusion.

\section{Fig. 2.}

A scatterplot of laser Doppler flowmetry (LDF)-based concentration-velocity slope values against laser speckle contrast imaging (LSCI)-LDF perfusion slope values. These data suggest that $\mathrm{LSCl}$ becomes less sensitive to changes in SkBF when these changes are more dependent on red blood cell concentration than red blood cell velocity.

\section{Fig. 3.}

Speckle contrast $(\sigma /<\mid>)$ as a function of $\mathrm{T}_{\mathrm{C}} / \mathrm{T}$, where $\mathrm{T}_{\mathrm{c}}$ is the correlation time (inversely proportional to velocity) and $T$ is the integration time, based on the algorithm of Fercher and Briers (1981) and assuming a Lorentzian velocity distribution. Note that the $\mathrm{x}$-axis uses a logarithmic scale. 